\title{
Vehicle Plate Recognition using Template Matching
}

\author{
${ }^{1}$ Nova Mayasari, ${ }^{2}$ Andysah Putera Utama Siahaan \\ Faculty of Science and Technology, Universitas Pembangunan Panca Budi, Medan, Indonesia \\ Email: ${ }^{1}$ maya7886@ pancabudi.ac.id, 2 andiesiahaan@gmail.com
}

\begin{abstract}
Vehicle plate is a marker of vehicle ownership. This plate is a unique number that cannot be the same for each vehicle. It consists of numbers and letters which represent or symbolize a particular area. Vehicle plate detection aims to find information related to the ownership of the vehicle. It is beneficial if applied to traffic intersections or to find information in the event of a traffic accident. The template matching method is a suitable method used to check vehicle plate patterns with data already stored in the vehicle database. The use of this method is excellent in helping to reduce crime and traffic accidents that often occur. If the system is well established, then people will not carelessly commit crime or hit and run on the road because the speed of vehicle license recognition is faster than the speed of someone to escape from the crime that just happened.
\end{abstract}

Key Words: Template Matching, Vehicle Plate, Pattern, Recognition

\section{INTRODUCTION:}

The introduction of vehicle license plate numbers is an important research area with many applications implementing it. Research on the introduction of vehicle license plate numbers has been carried out with certain advantages and disadvantages. It is because the vehicle license plate number represents a complex, so to develop an ideal computational model for vehicle license plate recognition is a very difficult thing [1]. Much data is obtained if the data observed displays a state that is by what we want. Therefore, things that relate to the shape, size, color, and other parameters that we all know because, in each element of the image, there are approximately two pieces of information about the location and color of the paint. The more information got from images or objects, the information about the shape, area, type, and model of an object and others [2]. It can also be used to obtain information from moving objects or images. Vehicle license number recognition techniques can be applied in several fields such as fields, commercial and security. These applications can be broadly classified into two groups such as some application inputs still use still images, while other applications use real-time dynamic images [3]. There are many recognition and pattern matching techniques for recording vehicle license plate numbers, one of which is the template matching method.

\section{THEORIES:}

\subsection{Computer Vision}

Computer vision has the primary goal to make useful decisions about real physical objects and scenes, based on images obtained from sensors [4]. Computer vision wants to make smart machines that can see [5]. Of course, this is not impossible. There are various examples of computer vision applications such as machines that monitor or examine millions of filaments from light bulbs or thousands of miles of factory fiber every day. ATMs have been built and are equipped with retina scans. The car can be driven by a computer using the camera as input [6]. Another terminology that is closely related to image processing is computer vision or machine vision. In essence, computer vision tries to imitate the workings of the human visual system (human vision). Human vision is very complicated. Humans see objects with their sense of sight (eyes), then the image of objects is passed on to the brain to be interpreted so that humans understand what objects appear in their eyes. The results of this interpretation may be used for decision making (for example avoiding seeing the car going ahead). Computer vision consists of techniques for estimating the characteristics of objects in the image to measure the characteristics associated with the geometry of the object, and interpreting the geometry information [7]. The processes in computer vision can be divided into three activities:

1. Obtain or acquire digital images.

2. Perform computational techniques to process or modify image data.

3. Analyzing and interpreting images and using processing results for specific purposes, such as guiding robots, controlling equipment, monitoring manufacturing processes, and others. 


\subsection{Image Processing}

An image is a visual image in two dimensions [8]. All operations to repair, analyze, or change an image are called image processing. The basic concept of the system of image processing is taken from the ability of the human sense of sight which is then linked to the ability of the human brain. In its history, image processing has been applied in various forms, with considerable success [9]. Like various other branches of science, image processing also involves various combinations of branches of science. Such as optics, electronics, mathematics, photography, and computer technology. Several factors cause the development of the image processing system to grow more rapidly today. One of the main things is the reduction in the cost of computer equipment needed. Both unit processing and bulk storage equipment are getting cheaper year by year. The second factor is the increasing availability of equipment for digital processing and image display. Various fields have used image processing applications in the commercial, industrial and medical fields. Image processing is an activity to improve image quality so that it can be easily interpreted by the system [10]. In general, the purpose of image processing is to transform or analyze an image so that new information about the image is made more explicit. Many ways can be applied in an image processing operation, almost mostly in optical form. Optical images are converted into electrical signals using video cameras or other similar devices. It converts the image representation of an optical light into a continuous electrical signal. This electrical signal is called an analog signal. Furthermore, analog images are digitized and transformed into digital data. Operations in image processing can be applied to an image with optical, analog, or digital shapes [11]-[13].

\section{METHODOLOGY:}

\subsection{Template Matching}

Template matching is a technique in digital image processing to find small parts of images that match the image template [14]. Template matching is one of the ideas used to explain how our brain recognizes forms or patterns. Templates in the context of pattern recognition refer to internal constructs that if matched with sensing stimuli lead to recognition of an object. Alternatively, pattern recognition occurs when there is a match between the sensory stimulus and the internal mental form [15]. This idea supports that a large number of templates have been created through our life experiences. Each template is related to a particular meaning. Template matching is a digital image processing method to compare between two similar objects by using small parts of an image that matches to the original image. Template matching is the process of looking for an object on an entire object in an image. The template is compared to the entire object and if the template matches (close enough) to an object that is not yet known in the image it is marked as a template [16]. Comparison between templates with all objects in the image can be done by calculating the difference in distance, as follows:

$$
D(m, n)=\sum \sum[f(j, k)-T(j-m, k-n)]
$$

The function $f(j, k)$ states the place image of the object to be compared to the template $T(j, k)$, while $D(m, n)$ states the distance between the template and the object in the image. In general, the template size is much smaller than the image size. Ideally, the template is said to match objects in the image if $\mathrm{D}(\mathrm{m}, \mathrm{n})=0$, but conditions like this are difficult to fulfill especially if the template is a grayscale image. Therefore, the rule used to declare a template matches an object is if $D(m, n)<L_{D}(m, n)$.

A primary template matching method uses convolution of objects that are adapted to certain features of image search, which is wanted to be detected. This technique can be quickly done on grayscale imagery or edge detection imagery. The highest convolution result is by the object structure where large object values can multiply large image values. This method is usually carried out by first selecting a part of image search to be used as an object: We will call the image search $\mathrm{S}(\mathrm{x}, \mathrm{y})$, where $(\mathrm{x}, \mathrm{y})$ is the coordinates of each image in image search. We will call the template $\mathrm{T}$ ( $\mathrm{xt}$, $\mathrm{yt}$ ), where (xt, yt) is the coordinates of each image in the template. We then just move the center (or origin) of the $\mathrm{T}$ template (xt, yt) over each $(\mathrm{x}, \mathrm{y})$ point in image search and calculate the number of products between the coefficients in $\mathrm{S}(\mathrm{x}, \mathrm{y})$ and $\mathrm{T}(\mathrm{xt}, \mathrm{yt})$ over the whole the area stretches by template. Because of all the possible positions of objects about image search, the position with the highest score is the best position. This method is sometimes referred to as 'Linear Spatial Filtering,' and the template is called a filter mask. For example, one way to handle translation problems in images, using template matching is to compare the intensity of the image, using the Sum of Absolute Differences (SAD (to measure. An image in the search for images with coordinates (xs, ys) has the intensity of Is (xs, ys) and the 
pixels in the template with coordinates (xt, yt) have an intensity of It (xt, yt), so the absolute difference in image intensity is defined as Diff (xs, ys, xt, yt) $=\mid$ Is (xs, ys) - It (xt, yt) $\mid$.

$$
S A D(x, y)=\sum \sum \operatorname{Diff}(x+i, y+j, i, j)
$$

\subsection{Vehicle Plate}

The data used is one of the first steps before data can be processed by the system more efficiently. This process will adjust the structure of the initial data into a structure that can be analyzed by the system, in the case of this study that is to convert data in the form of images into text. The data used includes object data and template data.

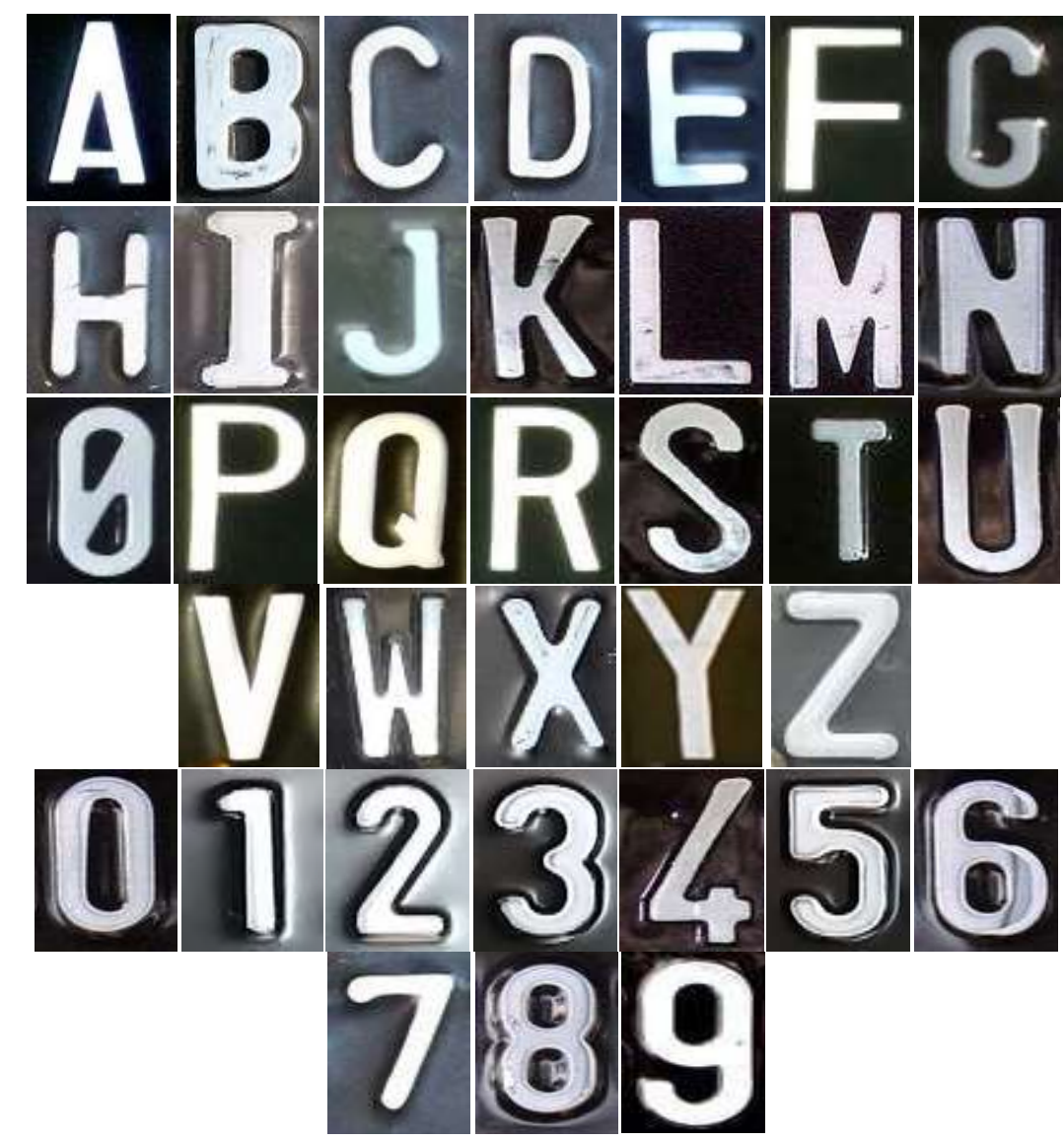

Figure 1. Pattern of vehicle number training for $A-Z$ and $0-9$

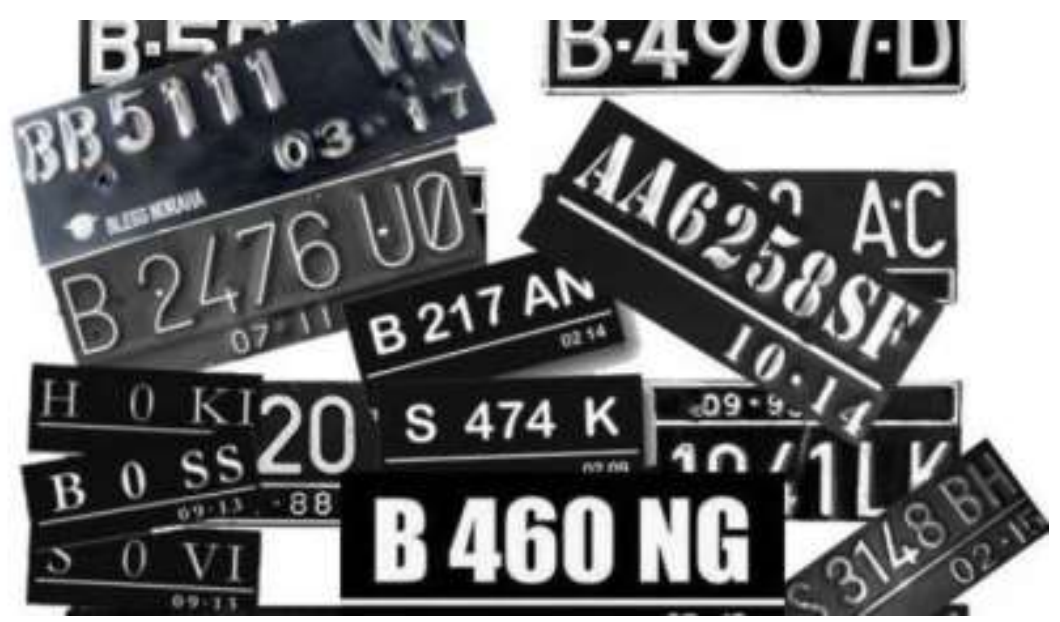

Figure 2. Examples of vehicle plates to be tested 
Figure 1 is the training formation model of the vehicle plate. Each letter of numerical funds will be collected to form 26 letters and ten numbers. These images will be trained to produce a low error value. Figure 2 is the plate that will be tested against the previously stored training data. Each plate will be broken down into letters and numbers which will then be compared with training data.

\section{RESULT AND DISCUSSION:}

The implementation of the use of the Template Matching algorithm is used to determine the classification of vehicle plates that have been previously trained. The input of vehicle plate that has been carried out by feature extraction process will be calculated the correlation value with each training data that has been trained previously. The test data that will be used is an image of a vehicle plate in the form of RGB images as many as 20 examples of randomly drawn images which are different and the same form and number from the training data. In each image that will be tested, will get an appropriate value or not depending on the ability of the Template Matching algorithm to do pattern recognition.

Table 1.

\begin{tabular}{|c|l|c|c|}
\hline No. & \multicolumn{1}{|c|}{ Plate } & Weight & Status \\
\hline 1 & BK 80 CA & 0,5508601920 & Yes \\
\hline 2 & BK 1234 UB & 0,5248468400 & Yes \\
\hline 3 & B 2476 UO & 0,4911983960 & Yes \\
\hline 4 & B 490 JD & 0,3618836900 & No \\
\hline 5 & B 460 NG & 0,4806943200 & Yes \\
\hline 6 & BB 5111 VK & 0,5865556500 & Yes \\
\hline 7 & AA 6258 SF & 0,5193294900 & Yes \\
\hline 8 & BK 1602 & 0,5028134680 & Yes \\
\hline 9 & BW 1874 FL & 0,6108075610 & Yes \\
\hline 10 & S 3148 BH & 0,4634386680 & No \\
\hline 11 & BK 1882 GJ & 0,4169213680 & No \\
\hline 12 & BK 1703 IE & 0,6388887120 & Yes \\
\hline 13 & BK 1574 IE & 0,6175932490 & Yes \\
\hline 14 & BK 265 PO & 0,7445287050 & Yes \\
\hline 15 & BK 1502 QQ & 0,6972607600 & Yes \\
\hline 16 & BL 923 ZU & 0,5481644900 & Yes \\
\hline 17 & H 10 KI & 0,4789748950 & No \\
\hline 18 & B 1041 LK & 0,4835884000 & Yes \\
\hline 19 & B 217 AN & 0,6496571730 & Yes \\
\hline 20 & BK 1745 TS & 0,4988596340 & Yes \\
\hline
\end{tabular}

Table 1 explains that the trial involved as many as 20 images. It shows that $85 \%$ of the training data tested show accurate results. It shows that pattern recognition with the template matching correlation method has a success rate and a reasonably high weight value ranging from 0.4 to 0.7 . It proves that this value shows that pattern recognition has good accuracy. The correlation between the matrix in the test image with the matrix on the training data has a great resemblance, while the correlation value below 0.5 has a correlation but not too good. Both matrices are said to correlate if the correlation value ranges between 0 and 1 . If the correlation value is 0 then there is no relationship between the two matrices, if the correlation value is 1 then there is a perfect positive relationship. If the correlation value is -1 there is a perfect negative linear relationship of the two matrices that are matched using the correlation algorithm and are said to be negatively correlated. It occurs when an increase in other variables follows an increase in one variable while a negative relationship is followed by an increase in one variable followed by a decrease in other variables. The higher the correlation value, the higher the similarity of the compared matrix. 


\section{CONCLUSION:}

The thing that is very influential in image processing is determining the position of the image during training and looking for weight values. This result will affect the results of the next process, and the results determine the suitability of vehicle license recognition. It will affect the accuracy of pattern recognition. Template Matching algorithm is a simple algorithm and performs well in recognizing an image pattern with a high success rate. This algorithm matches each pixel of the test data with the training data. If there are differences regarding the position or type of letter, it will affect the results of the weight value. The weight value ranges from 0 to 1 , the higher the weight value, the higher the chance of the similarity between the two images.

\section{REFERENCES:}

1. C.-H. Chen, T.-Y. Chen, M.-T. Wu, T.-T. Tang, and W.-C. Hu, "License Plate Recognition for Moving Vehicles Using a Moving Camera," in 2013 Ninth International Conference on Intelligent Information Hiding and Multimedia Signal Processing, 2013, pp. 497-500.

2. Chuin-Mu Wang and Jian-Hong Liu, "License plate recognition system," in 2015 12th International Conference on Fuzzy Systems and Knowledge Discovery (FSKD), 2015, pp. 1708-1710.

3. S.-L. Chang, L.-S. Chen, Y.-C. Chung, and S.-W. Chen, "Automatic License Plate Recognition," IEEE Trans. Intell. Transp. Syst., vol. 5, no. 1, pp. 42-53, Mar. 2004.

4. S. Caraiman et al., "Computer Vision for the Visually Impaired: the Sound of Vision System," in 2017 IEEE International Conference on Computer Vision Workshops (ICCVW), 2017, pp. 1480-1489.

5. J. Kim, J. K. Lee, and K. M. Lee, "Accurate Image Super-Resolution Using Very Deep Convolutional Networks," in 2016 IEEE Conference on Computer Vision and Pattern Recognition (CVPR), 2016, pp. 16461654.

6. T. Zielke, M. Brauchkmann, and W. von Seelen, "CARTRACK: computer vision-based car following," in [1992] Proceedings IEEE Workshop on Applications of Computer Vision, pp. 156-163.

7. C.-Y. Lin, W.-W. Chang, and Y.-H. Chen, "Intelligent Projector System Based on Computer Vision," in 2011 Fifth International Conference on Genetic and Evolutionary Computing, 2011, pp. 176-179.

8. A. P. U. Siahaan, "RC4 Technique in Visual Cryptography RGB Image Encryption."

9. W. Fitriani, R. Rahim, B. Oktaviana, and A. P. U. Siahaan, "Vernam Encypted Text in End of File Hiding Steganography Technique,” Int. J. Recent Trends Eng. Res., vol. 3, no. 7, pp. 214-219, Jul. 2017.

10. M. D. L. Siahaan and A. P. U. Siahaan, "Fingerprint Pattern Recoqnition Using LVQ," IOSR J. Comput. Eng., vol. 18, no. 6, pp. 85-92, 2016.

11. A. P. U. Siahaan, "Various Patterns of Data Mining Techniques."

12. G. Gunawan et al., "Mobile Application Detection of Road Damage using Canny Algorithm," J. Phys. Conf. Ser., vol. 1019, p. 012035, Jun. 2018.

13. A. P. U. Siahaan, "High Complexity Bit-Plane Security Enchancement in BPCS Steganography," Int. J. Comput. Appl., vol. 148, no. 3, pp. 17-22, 2016.

14. F. Essannouni and D. Aboutajdine, "Fast Frequency Template Matching Using Higher Order Statistics," IEEE Trans. Image Process., vol. 19, no. 3, pp. 826-830, Mar. 2010.

15. P. K. Lim et al., "Adaptive template matching of photoplethysmogram pulses to detect motion artefact," Physiol. Meas., vol. 39, no. 10, p. 105005, Oct. 2018.

16. S. Nikan and M. Ahmadi, "Partial Face Recognition Based on Template Matching," in 2015 11th International Conference on Signal-Image Technology \& Internet-Based Systems (SITIS), 2015, pp. 160-163. 